\title{
Refining Tabular Parsers for TAGs
}

\author{
Éric Villemonte de la Clergerie \\ INRIA, \\ Domaine de Voluceau \\ Rocquencourt, B.P. 105, 78153 Le Chesnay (France) \\ Eric.De_La_Clergerie@inria.fr
}

\begin{abstract}
This paper investigates several refinements of a generic tabular parser for Tree Adjoining Grammars. The resulting parser is simpler and more efficient in practice, even though the worst case complexity is not optimal.
\end{abstract}

\section{Introduction}

In (Villemonte de la Clergerie and Alonso Pardo, 1998), we have presented a generic Dynamic Programming [DP] interpretation for 2-Stack Automata [2SA]. These automata may be used to describe various kinds of parsing strategies for Tree Adjoining Grammars [TAG] and Linear Indexed Grammars [LIG], while the DP interpretation ensures the existence of tabular parsers satisfying the prefix-valid property and running in (optimal) worst-case time complexity $O\left(n^{6}\right)$ and space complexity $O\left(n^{5}\right)$ where $n$ is the length of the input string. ${ }^{1}$ An implementation, extended to handle unification, has been realized within DyALog system and tested for Feature TAGs (Alonso Pardo et al., 2000).

However, our analysis of that work was that the DP interpretation is relatively complex and the results not convincing. We believe that one reason for that is that the original DP interpretation was designed for 2SAs in general and does not take into account the particularities of TAGs. By analyzing how information is used when parsing TAGs, we are able to weaken the original DP interpretation. The new interpretation, while not ensuring optimal worst-case complexity w.r.t. the length of the input string (but still a polynomial one), has shown better performances for realistic linguistic grammars and sentences.

The paper begins, in Section 2, with a brief introduction to TAGs. Section 3 shows how parsing TAGs may be described using a 2-stack automaton. Section 4 presents the original "strong" DP interpretation. Section 5 shows how a "weaker" DP interpretation may be derived. Results are provided in Section 6 .

\footnotetext{
${ }^{1}$ Note that the optimal worst-case space complexity for non prefix-valid parsers is $O\left(n^{4}\right)$.
}

\section{Tree Adjoining Grammars}

A Tree Adjoining Grammar [TAG] (Joshi, 1987) is given by a set of elementary trees partitioned into initial trees and auxiliary trees. A node $\nu$ is labeled by $l_{\nu}$, which is a non-terminal for internal nodes and a terminal or a non-terminal for leaves. Each auxiliary tree $\beta$ has a distinguished leaf, called its foot and labeled by a non-terminal, the same as the root node of $\beta$.
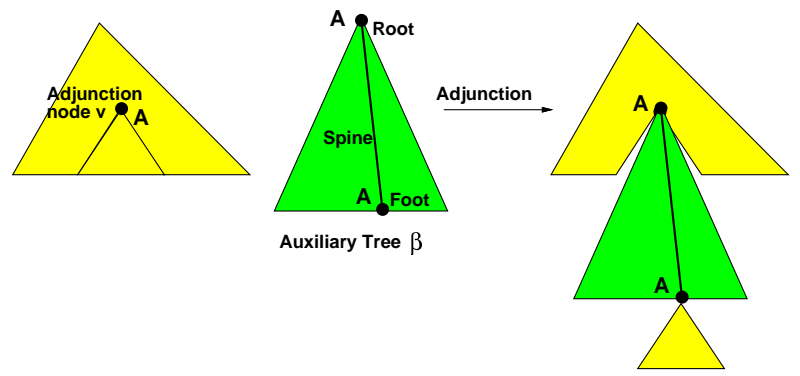

Figure 1: Adjunction

Two operations may be used to derive trees from elementary trees. The first one, called substitution, replaces a leaf node labeled by a non-terminal $A$ by an initial tree $\alpha$ whose root is also labeled by $A$. The second operation, called adjunction, is illustrated by Figure 1. An auxiliary tree $\beta$ whose root is labeled by $A$ may be adjoined at any node $\nu$ labeled by $A$. The subtree rooted at $\nu$ (and noted $t_{\nu}$ ) is grafted to the foot of $\beta$. Adjunctions occurring on a spine lead to nested adjunction.

Feature TAGs extend TAGs by attaching to nodes a pair of top and bottom arguments, represented by first-order terms.

\section{Describing parsing strategies with 2SAs}

Parsing TAGs may be viewed as traversing elementary trees from left to right $^{2}$ with suspensions occurring on substitution and adjunction nodes.

\footnotetext{
${ }^{2}$ Of course, other kinds of traversal are possible, such as right to left or bidirectional ones.
} 
As illustrated in Figure 2, we associate to each node $\nu$ the following information, in order to describe a top-down prefix-valid ${ }^{3}$ left-to-right parsing strategy :

- left and right position variables ${ }^{\circ} \mathrm{P}_{\nu}$ and $\mathrm{P}_{\nu} \cdot$ (resp.. $\mathrm{P}_{\nu}$ and $\mathrm{P}_{\nu \bullet}$ ) delimiting the span of $\nu$, possibly with adjunction (resp. without adjunction). When no adjunction is done on $\nu$, we will have to ensure that ${ }^{\bullet} \mathrm{P}_{\nu}={ }_{\bullet} \mathrm{P}_{\nu}$ and $\mathrm{P}_{\nu \bullet}=\mathrm{P}_{\nu}^{\bullet}$.

- dotted nodes ${ }^{\bullet} \nu$ and $\nu^{\bullet}\left(\operatorname{resp.} . \nu\right.$ and $\left.\nu_{\bullet}\right)$ denoting computation points associated to entering and exiting $\nu$, possibly with adjunction (resp. without adjunction). As shown in Figure 2, each dotted node is implicitly associated to a position variable.

- call and return terms ${ }^{\bullet} \mathrm{T}_{\nu}=l_{\nu}\left({ }^{\bullet} \mathrm{P}_{\nu}\right)$ and $\mathrm{T}_{\nu}^{\bullet}=\operatorname{ret}\left(\mathrm{P}_{\nu}^{\bullet}\right)\left(\right.$ resp. $\bullet^{\circ} \mathrm{B}_{\nu}=l_{\nu}\left(\bullet^{\mathrm{P}_{\nu}}\right)$ and $\left.\mathrm{B}_{\nu_{\bullet}}=\operatorname{ret}\left(\mathrm{P}_{\nu_{\bullet}}\right)\right)$ used as pieces of information when starting or resuming a suspension for the node, possibly with adjunction (resp. without adjunction). ${ }^{4}$

Note that there are many implicit congruences between positions variables and dotted nodes. For instance, for a node $\mu$ visited just after $\nu$, we have $\nu^{\bullet} \equiv{ }^{\bullet} \mu$ and $\mathrm{P}_{\nu}{ }^{\bullet} \equiv{ }^{\bullet} \mathrm{P}_{\mu}$.

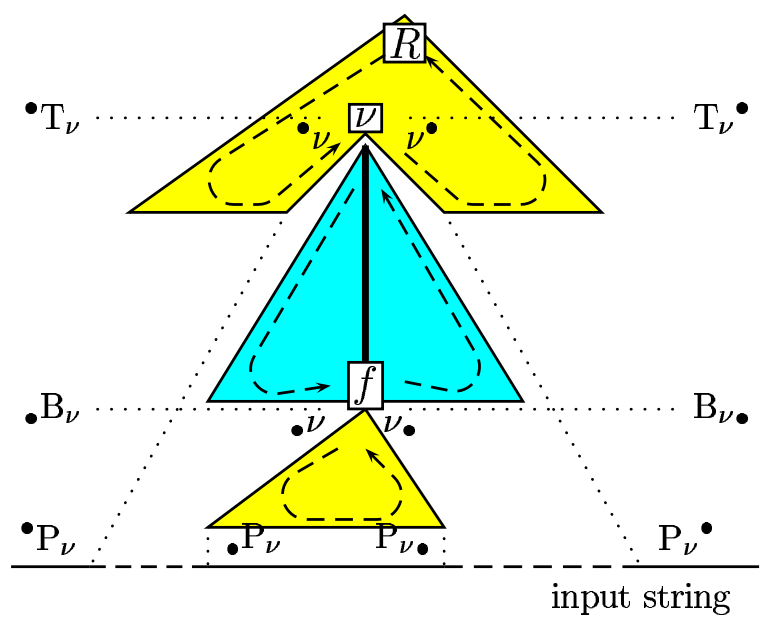

Figure 2: Some notations for TAGs

In order to simplify notation and because the case of adjunction will come again and again, we also omit most subscripts when possible, assuming the following equalities (for a successful adjunction of

\footnotetext{
${ }^{3}$ By prefix-valid we mean that failure in an alternative is detected as soon as a parsed prefix of the input string has no way to be completed by some string into a valid parse.

${ }^{4}$ These terms arise from the modulated Call/Return model(Alonso Pardo et al., 2000). Other values are possible for other parsing strategies, in particular when dealing with Feature TAGs.
}

auxiliary tree $\beta$ at node $\nu$ ).

$$
\begin{aligned}
& \bullet{ }^{\bullet} \equiv{ }^{\bullet} \mathrm{T}_{\nu} \equiv{ }^{\bullet} \mathrm{T}_{\mathrm{r}_{\beta}} \quad \mathrm{T}^{\bullet} \equiv \mathrm{T}_{\nu}{ }^{\bullet} \equiv \mathrm{T}_{\mathrm{r}_{\beta}} \bullet \\
& \bullet \mathrm{B} \equiv \bullet_{\bullet} \mathrm{B}_{\nu} \equiv{ }_{\bullet} \mathrm{B}_{\mathrm{f}_{\beta}} \quad \mathrm{B}_{\bullet} \equiv \mathrm{B}_{\nu \bullet} \equiv \mathrm{B}_{\mathrm{f}_{\beta}}
\end{aligned}
$$

Intuitively, when reaching the entry point ${ }^{\bullet} \nu$ with left position ${ }^{\circ} \mathrm{P}_{\nu}$ and deciding to perform an adjunction, the current traversal is suspended and the call information ${ }^{-} \mathrm{T}_{\nu}$ is used to fetch some auxiliary tree $\beta$ and to start its traversal. When reaching foot $f_{\beta}$, the traversal of $\beta$ is suspended and ${ }^{\circ} \nu$ is resumed and leads to $\bullet \nu$ at position ${ }^{\circ} \mathrm{P}_{\nu}$. When reaching $\nu_{\bullet}$ at position $\mathrm{P}_{\nu \bullet}$ (denoting the recognition of $\nu$ without adjunction), a new suspension occurs and the traversal of $\beta$ is resumed on the left of its spine. When the traversal of $\beta$ is finished, $\nu_{\bullet}$ is resumed and leads to $\nu^{\bullet}$ at position $\mathrm{P}_{\nu}{ }^{\bullet}$ (denoting the recognition of $\nu$ with adjunction).

As illustrated by Figure 3, these various steps may naturally be described using two stacks, a master stack MS used to record the tree traversals and an auxiliary stack $\mathbf{A S}$ used to keep the information needed to resume suspensions due to adjunctions. We have chosen to take $\bar{\nu}=\nu$ (the name of the node) and $\underline{\nu}=\perp$ (the null information) as pieces of information recorded on AS, but other choices are possible for other parsing strategies. ${ }^{5}$ In Figure 3, we also use composite stack elements of the form . $\mathrm{B}_{\nu}+\bar{\nu}$ (transition FCALL) to compose information relative to a node $\left(. \mathrm{B}_{\nu} \equiv{ } \mathrm{B}_{\mathrm{f}_{\beta}}\right.$ for the footnode $f_{\beta}$ ) and information relative to an adjunction ( $\bar{\nu}$ for the adjunction node $\nu$ ).

More formally, the parsing steps may be expressed as transitions of a 2-stack automaton (Becker, 1994; Villemonte de la Clergerie and Alonso Pardo, 1998). A transition $\tau$ of the form $(\Xi, \xi) \longmapsto(\Theta, \theta)$ applies on any configuration (MS, AS $)=(\Psi \Xi, \psi \xi)$ and returns $(\Psi \Theta, \psi \theta)$. Some of the steps of the parsing strategies are explicited by the following transitions, with $X, Y$ denoting free variables, instantiated by matching during transition application. ${ }^{6}$

$$
\begin{array}{ll}
\text { ACALL } & (\bullet \nu, \epsilon) \longmapsto\left(\bullet \nu \bullet \mathrm{T}_{\nu}, \bar{\nu}\right) \\
\text { ARET } & \left.\left(\bullet \nu \mathrm{T}_{\nu}, \underline{\nu}\right) \longmapsto(\nu), \epsilon\right) \\
\text { ASEL } & \left(\bullet \mathrm{T}_{\mathrm{r}}, \epsilon\right) \longmapsto(\bullet \mathrm{r}, \epsilon) \\
\text { APUB } & \left(\mathrm{r}^{\bullet}, \epsilon\right) \longmapsto\left(\mathrm{T}_{\mathrm{r}}^{\bullet}, \epsilon\right) \\
\text { FCALL } & (\bullet \mathrm{f}, X) \longmapsto\left(\bullet \mathrm{f}\left(\bullet \mathrm{B}_{\mathrm{f}}+X\right), \epsilon\right) \\
\text { FRET } & \left.\left(\bullet \mathrm{B}_{\mathrm{f} \bullet}+Y\right), \epsilon\right) \longmapsto(\mathrm{f} \bullet, Y) \\
\text { FSEL } & \left(\bullet \mathrm{B}_{\nu}+\bar{\nu}, \epsilon\right) \longmapsto(\bullet \nu, \epsilon) \\
\text { FPUB } & (\nu \bullet, \epsilon) \longmapsto\left(\left(\mathrm{B}_{\nu \bullet}+\underline{\nu}\right), \epsilon\right)
\end{array}
$$

\footnotetext{
${ }^{5}$ For instance, we may handle adjunctions in a bottom-up way by taking $\bar{\nu}=\perp$ and $\underline{\nu}=\nu$.

${ }^{6}$ Actually, the basic model of 2SA uses symbols as stack elements and explicits the propagation of the position variables as well as of other pieces of information. An extended model uses terms as stack elements and uses unification to apply transitions and handle position variables and features. We are using this extended model but have decided to skip details in this presentation for sake of clarity and because they are not essential to the discussion.
} 


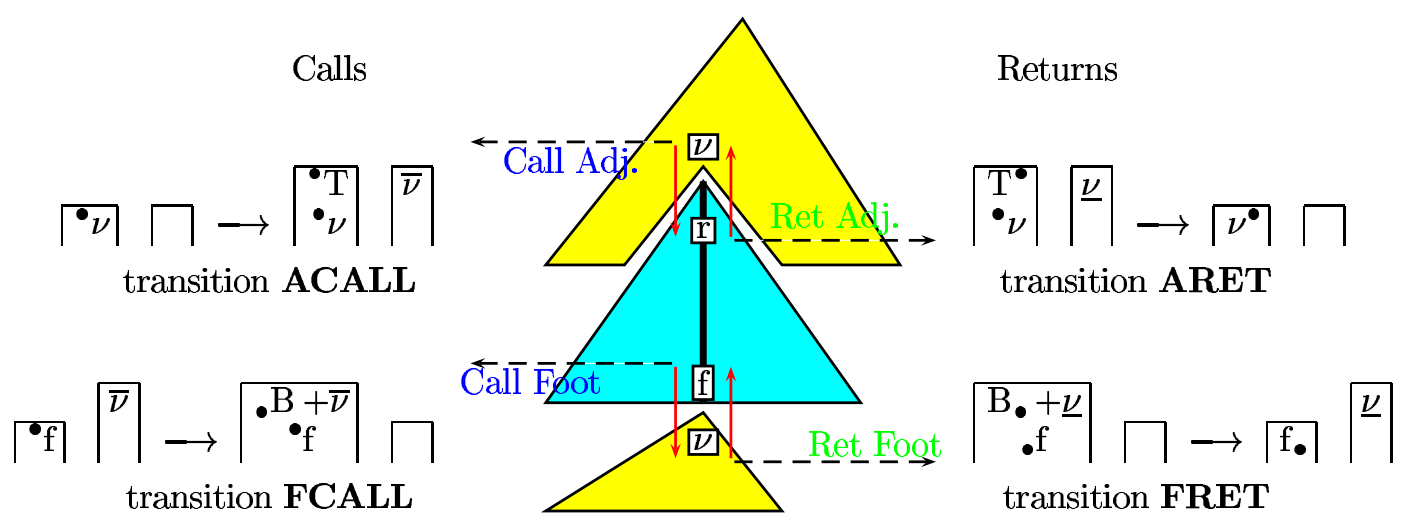

Figure 3: Illustration of some steps

In practice, the transitions may be grouped in meta-transitions by (a) grouping pairs of related call and return transitions such as ACALL and ARET and (b) considering dotted nodes such as $\nu^{\bullet}$ as continuations. For instance, figure 4 shows the skeleton of a meta-transition representing the traversal of an auxiliary tree with root $r$ and some adjoinable node $\nu{ }^{7}$

The typed feature-structure representation of the meta-transitions has no special signification, except that it closely corresponds to the internal representation used by our compiler. Meta-transitions are important for the rest of the paper, because they explicit some particularities of TAGs (and of the parsing strategy).

There is one meta-transition per tree, possibly including disjunctive values to handle non mandatory adjunction nodes. Actually, it is theoretically possible to merge into some disjunctive meta-transition the traversal of several trees. ${ }^{8}$

\section{Strong DP interpretation}

The strong Dynamic Programming interpretation of 2SAs is based on the identification of elementary 2SA derivations that may be reused and that may be represented by items. Application rules are provided to combine together items and transitions in order to retrieve all possible 2SA derivations. We just introduce minimal background about the interpretation and readers are invited to consult (Villemonte de la Clergerie and Alonso Pardo, 1998; Alonso Pardo et al., 2000).

The interpretation considers two kinds of items, namely Context-Free [CF] Items $\boldsymbol{A} / \boldsymbol{B} / \boldsymbol{C}$ (also represented by $\boldsymbol{A} / \boldsymbol{B} /\left[{ }_{\infty}\right] \boldsymbol{C}$ ) and Escaped Context-

\footnotetext{
${ }^{7}$ Actually, we have considered the case of a mandatory adjunction at $\nu$. To handle non mandatory ones, we add disjunction points in the meta-transitions and share common continuations between alternatives.

${ }^{8}$ There are relationships to investigate between the metatransitions and the finite-state automata used in (Evans and Weir, 1998; Carroll et al., 1998).
}

Free $[\mathrm{xCF}]$ Items $\boldsymbol{A} / \boldsymbol{B} /[\boldsymbol{D E}] \boldsymbol{C}$ representing subderivations passing by configurations $\mathcal{A}, \mathcal{B}, \mathcal{C}, \mathcal{D}$ and $\mathcal{E} .^{9}$ The items keep only projections of the configurations, and the less information is kept, the better computation sharing will potentially be. Practically, for a configuration $\mathcal{X}=(\Xi X, \xi x)$, only the topmost elements $X$ and $x$ of each stack need to be kept in a mini configuration $\langle X, x\rangle$ or a micro configuration $\langle X\rangle$. One can further reduce the amount of information by keeping for $X$ only the "triggering" fraction of information $\epsilon(X)$ that is consulted to start some computation above $X$ (on the stack MS).

\begin{tabular}{|c|c|c|c|c|}
\hline dotted node $X$ & ${ }^{\bullet} \nu$ & $\bullet \nu$ & $\nu_{\bullet}$ & $\nu^{\bullet}$ \\
\hline$\epsilon(X)$ & ${ }^{\bullet} \mathrm{T}_{\nu}$ & $\bullet \mathrm{B}_{\nu}$ & $\mathrm{B}_{\nu \bullet}$ & $\mathrm{T}_{\nu}$ \\
\hline
\end{tabular}

Finally, for TAGs, Table 1 displays the patterns for the various items related to an adjunction on node $\nu$ in a tree rooted at $r$. If $\nu$ dominates some foot $g$ in an adjunction on $\mu,[\boldsymbol{D E}]=\left[\left\langle\mathrm{B}_{\mathrm{g}}, a\right\rangle\left\langle\mathrm{B}_{\mathrm{g}}+b\right\rangle\right]$ and $(a, b)=(\bar{\mu}, \underline{\mu})$; otherwise $[\dot{\boldsymbol{D} E}]=[\diamond \diamond]$ and $(a, b)=(\diamond, \diamond)$.

There are several generic rules used to combine items and transitions, some of them being rather complex. More precisely, when parsing TAGs, the most complex case corresponds to returning from a fully recognized auxiliary tree $\beta$ to the adjunction node $\nu$. In first approximation, this step requires combining three items (start of the adjunction, recognition of the subtree $t_{\nu}$ and recognition of $\beta$ ) with a transition ARET. However, a straightforward application does not ensure an optimal worstcase time complexity in $O\left(n^{6}\right)$ and this step is actually split into the rules of Figure 5, with the introduction of an intermediary structure with superscript $L$.

Furthermore, still to ensure optimal worst-case complexity, projections of items have to be considered with some components removed (marked by $\star$ )

\footnotetext{
${ }^{9}$ The diverse conditions satisfied by these configurations are outside the scope of this paper.
} 


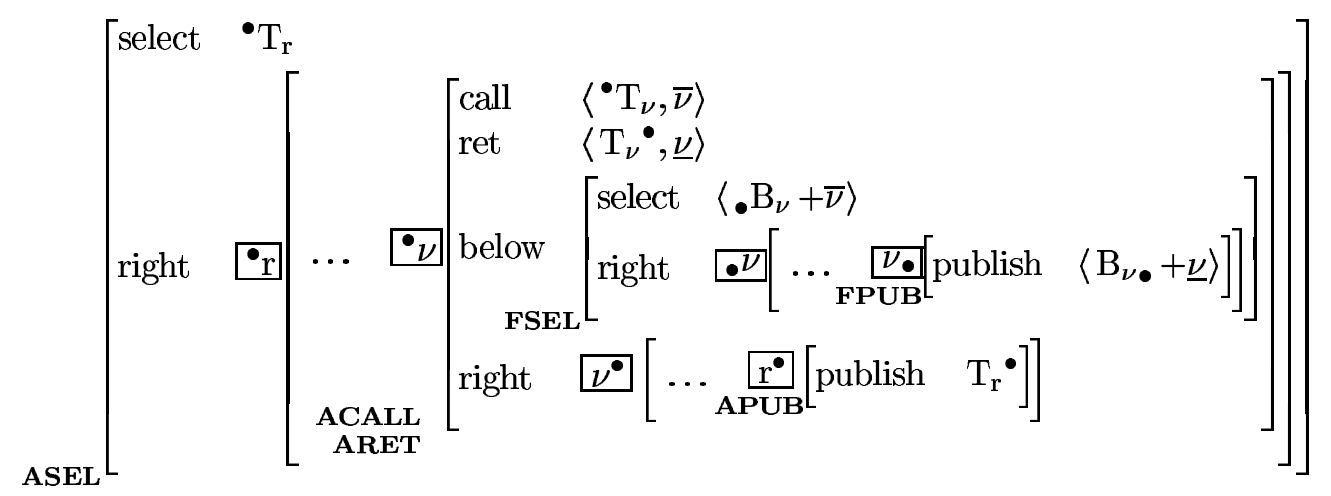

Figure 4: Sketch of a meta-transition for an auxiliary tree [strong interpretation]

\begin{tabular}{|l|l|l|}
\hline & after CALL & before RET \\
\hline on ADJ $\nu$ & $\left\langle^{\circ} \mathrm{T}\right\rangle /\left\langle{ }^{\circ} \mathrm{T}\right\rangle /\left\langle{ }^{\circ} \mathrm{T}, \bar{\nu}\right\rangle$ & $\left.\left\langle{ }^{\circ} \mathrm{T}\right\rangle /{ }^{\circ} \mathrm{T}\right\rangle /\left[\left\langle\bullet_{\bullet} \mathrm{B}, \bar{\nu}\right\rangle\langle\mathrm{B} \bullet+\underline{\nu}\rangle\right]\left\langle\mathrm{T}^{\bullet}, \underline{\nu}\right\rangle$ \\
\hline on FOOT $f$ & $\left\langle{ }^{\circ} \mathrm{T}_{\mathrm{r}}\right\rangle /\left\langle{ }_{\bullet} \mathrm{B}, \bar{\nu}\right\rangle /\left\langle{ }_{\bullet} \mathrm{B}+\bar{\nu}, a\right\rangle$ & $\left\langle{ }^{\circ} \mathrm{T}_{\mathrm{r}}\right\rangle /\left\langle{ }_{\bullet} \mathrm{B}, \bar{\nu}\right\rangle /[\boldsymbol{D E}]\left\langle\mathrm{B}_{\bullet}+\underline{\nu}, b\right\rangle$ \\
\hline
\end{tabular}

Table 1: Items at adjunction and foot nodes [strong interpretation]

in the rules. In particular, three projections are needed for the recognition of $t_{\nu}$, the subtree rooted at $\nu$, in the context of an adjunction on $\nu$.

For TAGs, these remarks lead to the execution model illustrated in Figure 6, obviously complex to decrypt ${ }^{10}$ and implement. This model also incorporates the notions of partial applications and immediate applications.

Partial applications decompose a complex application rule into a cascade of elementary applications, which combine only an item and a transition or some intermediary object (close to a transition). Immediate application is a (partial) application done as soon as a dotted node $X$ is reached, in order to avoid tabulating an item relative to $X$.

Immediate application is made possible because the transitions have been grouped into metatransitions. For instance, when reaching an adjunction node $\nu$ with dotted computation point $\bullet \nu$, one can immediately install:

- an item CAI to fetch and start the recognition of some auxiliary tree.

- an intermediary transition CFI > preparing the recognition of the subtree $t_{\nu}$ rooted at $\nu$.

- a projection item $I_{a}$ preparing the return from $t_{\nu}$ to the auxiliary tree.

- an intermediary transition RAI > preparing the return from the auxiliary tree to the adjunction node.

\footnotetext{
${ }^{10}$ To help the reading, object names follow the model Call/Return Aux/Foot (Item or transition $\mathbf{I}>$ ) plus indices for projections $(1,2,3, \ldots)$ and intermediary objects $(a, b$, $c \ldots)$, giving for instance CAI, $\mathrm{RFI}_{b}, \mathrm{RFI}>1, \ldots$
}

Looking at Figure 6, we observe the three projections $\mathrm{RFI}_{\{a, b, c\}}$ needed to record the recognition of subtree $t_{\nu}$ and the two complex cascades of (partial) applications required to return to the auxiliary tree and to the adjunction node.

\section{Weak DP interpretation}

The strong DP interpretation derives from a generic view of 2SAs, independently from TAGs. Efforts have been made to ensure an optimal worst-case complexity but they lead to a complex model. Furthermore, it is not proven that these efforts are worthwhile in practice.

Therefore, we tried to exploit the properties of TAGs to simplify the strong DP interpretation into two directions:

1. Removing more information from items.

2. Relaxing tabulation, possibly increasing worstcase complexity, but still ensuring polynomial worst-case complexity.

To remove information, we can note that parsing an auxiliary tree $\beta$ does not depend on the node $\nu$ that started the adjunction. The only pertinent information (needed at the root and foot nodes) is given by ${ }^{\bullet} \mathrm{T}, \mathrm{T}^{\bullet}, . \mathrm{B}$, and $\mathrm{B}_{\bullet}$. The node name $\nu$ is needed only when leaving the foot node to parse the subtree $t_{\nu}$ rooted at $\nu$. But for TAGs, the meta-transition indicates, as soon as $\boldsymbol{\nu}^{\nu}$ is reached, the continuation $\bullet \nu$ to follow. By immediate application, one can prepare an intermediary transition waiting for a compatible foot node to be reached. Practically, this idea means that $\bar{\nu}$ and $\underline{\nu}$ may be removed and that we can use micro configurations $\langle X\rangle$ 
$(\mathrm{dxCF})$

$(\mathrm{dxCF})$

(ARET)

$$
\begin{aligned}
& \begin{array}{l}
\star /\left\langle_{\bullet} \mathrm{B}, \bar{\nu}\right\rangle /[\boldsymbol{D E}]\left\langle\mathrm{B}_{\bullet}+\underline{\nu}, \star\right\rangle^{\mathrm{RFI}_{b}} \\
\left\langle{ }^{\bullet} \mathrm{T}\right\rangle /\left\langle{ }^{\bullet} \mathrm{T}\right\rangle /[\langle\bullet \mathrm{B}, a\rangle\langle\mathrm{B} \bullet+\underline{\nu}\rangle]\left\langle\mathrm{T}^{\bullet}\right\rangle^{\mathrm{RAI}}
\end{array} \\
& \left\langle{ }^{\bullet} \mathrm{T}_{\mathrm{r}}\right\rangle / \boldsymbol{N} /\left\langle{ }^{\bullet} \nu, a\right\rangle^{I} \\
& \left\langle\bullet^{\bullet} \mathrm{T}_{\mathrm{r}}\right\rangle / \star /[\boldsymbol{D E}]\langle\star, b\rangle^{\mathrm{RFI}_{c}} \\
& \frac{\left({ }^{\bullet} \nu \mathrm{T}^{\bullet}, \underline{\nu}\right) \longmapsto\left(\nu^{\bullet}, \epsilon\right) \quad\left\langle\bullet^{\bullet}\right\rangle\left\langle{ }^{\bullet} \mathrm{T}\right\rangle[[\boldsymbol{D E}]]\left\langle\mathrm{T}^{\bullet}, \underline{\nu}\right\rangle^{L}}{\left\langle\mathrm{~T}_{\mathrm{r}}\right\rangle / \boldsymbol{N} /[\boldsymbol{D E}]\left\langle\nu^{\bullet}, b\right\rangle} \\
& \boldsymbol{D}=\left\langle\bullet \mathrm{B}_{\mathrm{f}}, a\right\rangle
\end{aligned}
$$

Figure 5: Some application rules [strong interpretation]

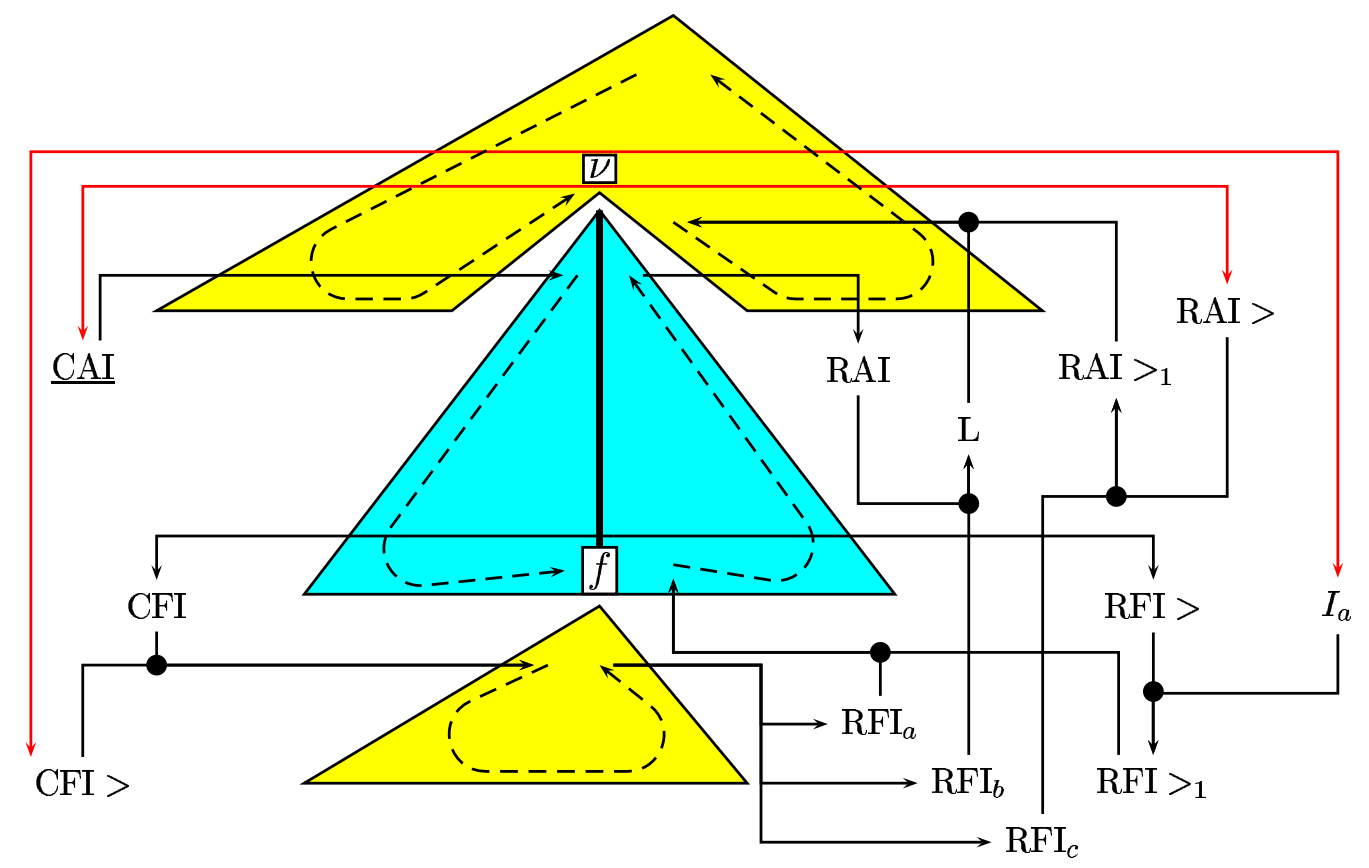

Figure 6: Cascades of partial evaluations related to an adjunction [strong interpretation]

in place of mini ones $\langle X, x\rangle$. We can also simplify composite terms $X+x$ into $X$.

Tabulation may be relaxed by changing the way the subtree $t_{\nu}$ is traversed. The strong DP interpretation traverses $t_{\nu}$ independently of the adjunctions that have been started (but not yet finished) for nodes on the path $\pi_{\nu}$ from $\nu$ to the root of $\alpha$ (if $\nu$ belongs to $\alpha$ ), hence allowing to share the traversal of $t_{\nu}$ for different adjunctions on $\nu$ starting at the same position but occurring in different contexts. In a way, the strong DP interpretation does not consider $t_{\nu}$ as a subtree of $\alpha$ but rather as a tree of its own. We decided to relax this condition by requiring that the traversal of $t_{\nu}$ be done in the context of a larger traversal of $\alpha$, including in particular the ongoing adjunctions on $\pi_{\nu}$.

Here again, this choice is possible because, when reaching dotted node $\nu_{\bullet}$ at the end of $t_{\nu}$, we know of course that we must suspend the computation (to finish traversing $\beta$ ) but also that, when resuming the computation, we can directly jump to $\nu^{\bullet}$. This difference of point of view leads to a revised version of meta-transitions, as illustrated by Figure 7 where $\nu^{\bullet}$ is now seen as the next continuation of $\nu_{\bullet}$ (to be compared with Figure 4 for dotted nodes $\bullet \nu$, and $\left.\nu_{\bullet}\right)$. The new meta-transition does not correspond to a set of new transitions but rather to a different organization of the original set.

Applying these various ideas leads to $\mathrm{CF}$ items $\boldsymbol{A} / \mathcal{N} / \boldsymbol{C}$ and xCF items $\boldsymbol{A} / \mathcal{N} /[\boldsymbol{D E}] \boldsymbol{C}$, where $\mathcal{N}$ denotes a stack of pairs $\left\langle{ }^{\bullet} \mathrm{T}_{\mu}\right\rangle\left\langle{ }_{\bullet} \mathrm{B}_{\mu}\right\rangle$. We can show that $\boldsymbol{A}=\left\langle{ }^{\bullet} \mathrm{T}_{\mathrm{r}}\right\rangle$ where $r$ is the root of some elementary tree $\alpha$ and $\boldsymbol{C}$ is some dotted node or information relative to a node $\nu$ of $\alpha$. The micro configuration $\left\langle{ }^{\bullet} \mathrm{T}_{\mu}\right\rangle$ records starting an adjunction at $\mu$ and leaving $\alpha$ while $\left\langle. \mathrm{B}_{\mu}\right\rangle$ records leaving a foot node and coming back to $\alpha$. Therefore, an item records the list of all adjunctions that are still under completion on $\pi_{\nu}$ for some node $\nu$ and tree $\alpha$. Table 2 displays 


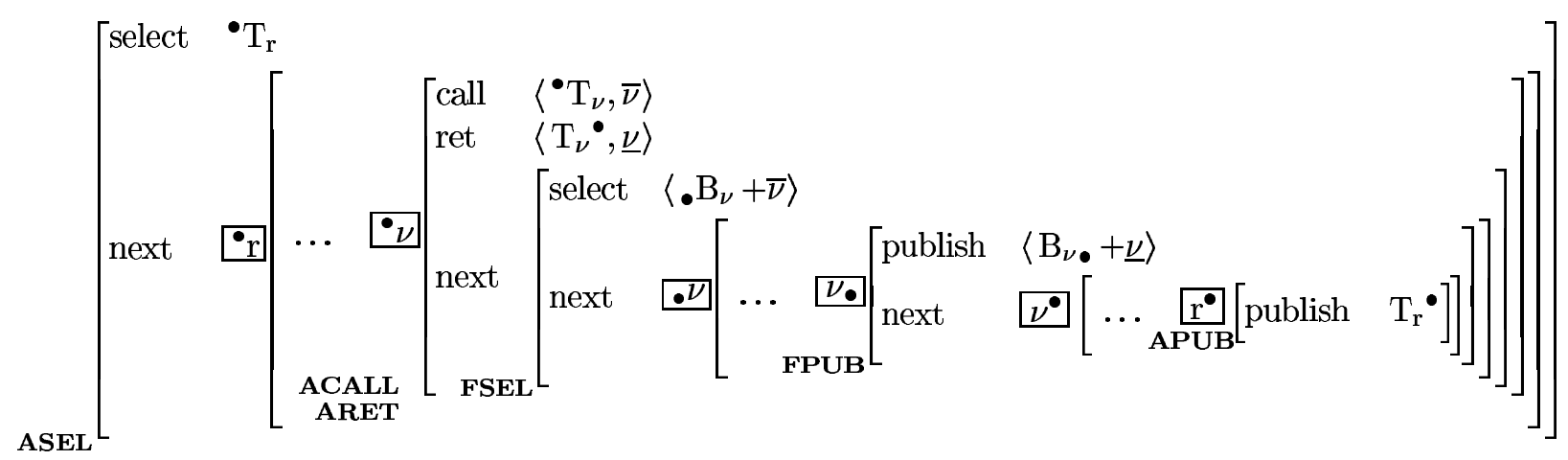

Figure 7: Sketch of a meta-transition [weak interpretation]

different patterns for weak items (to be compared with Table 1).

Some of the application rules for the weak DP interpretation are provided in Figure 8 and are illustrated by the execution model of Figure 9. Clearly, the execution model is simpler than the one for the strong DP interpretation (Figure 6).

Analyzing worst-case complexity, we find that it remains polynomial but now depends on the maximal depth $d$ of elementary trees. More precisely, worst-case complexities are $O\left(n^{5+2 d}\right)$ for time $e^{11}$ and $O\left(n^{4+2 d}\right)$ for space. These complexities suggest that the weak DP interpretation is maybe not very efficient for deep elementary trees.

We can also analyze worst-case complexities w.r.t. the size $G$ of the grammar (given for instance by the number of elementary trees). We find space and time complexities in $O\left(G^{2}\right)$ for the strong DP interpretation and in $O(G)$ for the weak one (because of the removing of $\bar{\nu}$ and $\underline{\nu}$ from items). More precisely, each item provides information about nodes on some path of a single elementary tree and while some application rules (say rule [ARET]) combine items relative to two different trees, one easily checks that the information of one of the item $(R A I)$ is fully constrained by the information coming from the other item and the transition $\left(K\right.$ and $\left.\mathrm{T}^{\bullet}=\epsilon\left(\nu^{\bullet}\right)\right)$. These complexities suggest that the weak interpretation is useful for large grammars, which is generally the case for linguistic grammars.

\section{Results}

We have modified the original implementation in DyALog $^{12}$ to encode the weak DP interpretation. We have then used it on a small Feature TAG for French of about 50 trees, organized following the XTAG architecture (The XTAG Research

\footnotetext{
${ }^{11}$ by counting the number of distinct string indices involved in [ARET] with items $K$ and $R A I$ with the fact that $\bullet \mathrm{B}$ and B. share their index.

${ }^{12}$ The system is freely available at http://atoll.inria. $\mathrm{fr} / \sim \mathrm{clerger}$ and the compiler may be modified to test new interpretations.
}

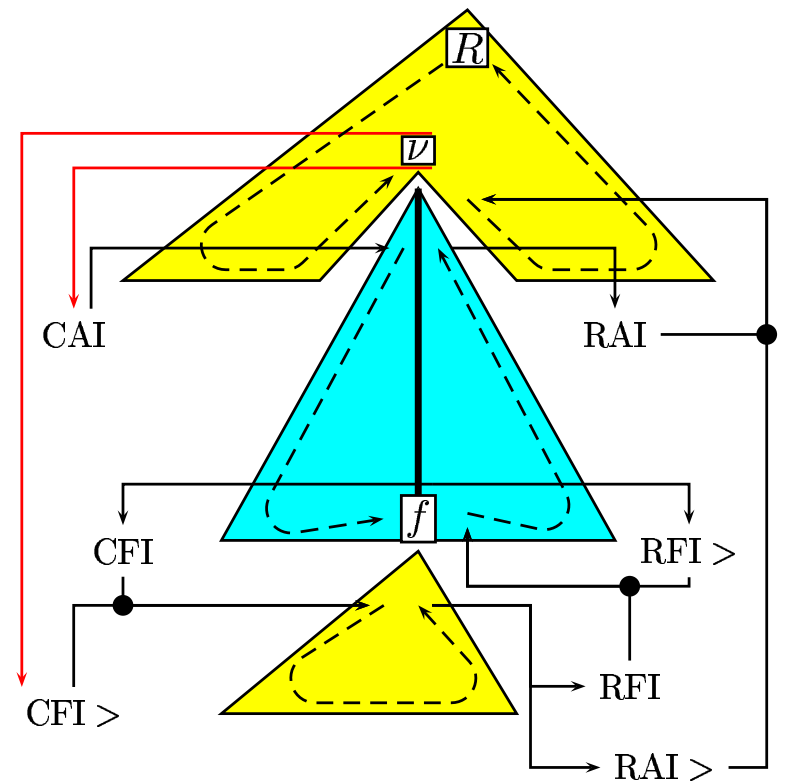

Figure 9: Execution model [weak interpretation]

Group, 1995). We have built and compared different parsers ${ }^{13}$, for the strong and weak DP interpretations but also for two different parsing strategies. The strategy TD corresponds to the top-down prefix-valid strategy mentioned in this paper, while the strategy Hybrid uses top-down prediction on the syntactic categories but bottom-up checking on the node feature arguments. Note that we do not assume the grammar to be lexicalized and we do not use any notion of lexicalization or tree filtering to speed-up parsing (Alonso Pardo et al., 2000).

The results of these experiments (Table 3) clearly show the efficiency of the weak DP interpretation for standard sentences and for a small but realistic linguistic grammar. In this table, the line $\emptyset$ indicates the loading time of each parser, time that we have subtracted from the running time for the sentences.

\footnotetext{
${ }^{13}$ They can be tried on line at http://medoc.inria.fr/ pub-cgi-bin/parser.cgi.
} 


\begin{tabular}{|l|l|l|}
\hline & after CALL & before RET \\
\hline on ADJ $\nu$ & $\left.\left\langle^{\circ} \mathrm{T}\right\rangle / /{ }^{\bullet} \mathrm{T}\right\rangle$ & $\left\langle{ }^{\bullet} \mathrm{T}\right\rangle / /\left[\left\langle\bullet_{\bullet} \mathrm{B}\right\rangle\left\langle\mathrm{B}_{\bullet}\right\rangle\right]\left\langle\mathrm{T}^{\bullet}\right\rangle$ \\
\hline on FOOT $f$ & $\left\langle^{\bullet} \mathrm{T}_{\mathrm{r}}\right\rangle / \mathcal{N}\left\langle{ }^{\bullet} \mathrm{T}\right\rangle\left\langle{ }_{\bullet} \mathrm{B}\right\rangle /\left\langle\iota_{\bullet} \mathrm{B}\right\rangle$ & $\left\langle{ }^{\bullet} \mathrm{T}_{\mathrm{r}}\right\rangle / \mathcal{N}\left\langle{ }^{\bullet} \mathrm{T}\right\rangle\left\langle\bullet{ }_{\bullet} \mathrm{B}\right\rangle /[\boldsymbol{D E}]\left\langle\mathrm{B}_{\bullet}\right\rangle$ \\
\hline
\end{tabular}

Table 2: Items at adjunction and foot nodes [weak interpretation]

(ACALL)

(FCALL)

$$
\begin{aligned}
& \frac{(\bullet \nu, \epsilon) \longmapsto\left(\bullet^{\bullet}{ }^{\bullet} \mathrm{T}, \bullet \nu\right) \quad \boldsymbol{A} / \star /\left\langle\bullet^{\bullet} \nu\right\rangle^{I}}{\left\langle{ }^{\bullet} \mathrm{T}\right\rangle / /\left\langle^{\bullet} \mathrm{T}\right\rangle^{\mathrm{CAI}}} \\
& \begin{array}{c}
A / \mathcal{N} /\left\langle\bullet^{\bullet} \nu\right\rangle^{I} \\
(. \mathrm{f}, \bullet \nu) \longmapsto(. \mathrm{f} \bullet \nu, \epsilon) \quad\left\langle{ }^{\bullet} \mathrm{T}\right\rangle / \star /\left\langle{ }^{\bullet} \mathrm{B}\right\rangle^{C F I} \\
\hline \boldsymbol{A} / \mathcal{N}\left\langle{ }^{\bullet} \mathrm{T}\right\rangle\langle\cdot \mathrm{B}\rangle /\langle\bullet \nu\rangle
\end{array} \\
& \text { CFI }>=I+\text { FCALL } \\
& \left\langle{ }^{\bullet} \mathrm{T}\right\rangle / \mathcal{O} /\langle\bullet \mathrm{f}\rangle^{J}
\end{aligned}
$$

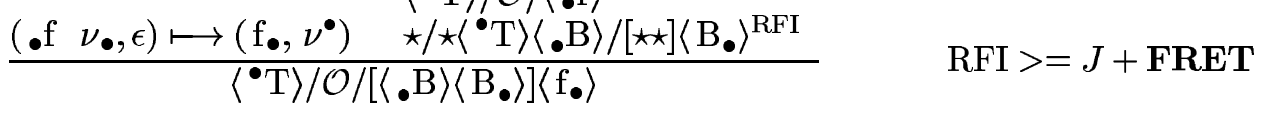

$$
\begin{aligned}
& \frac{\left(\bullet \nu \mathrm{T}^{\bullet}, \nu^{\bullet}\right) \longmapsto\left(\nu^{\bullet}, \epsilon\right) \quad \begin{array}{l}
\boldsymbol{A} / \mathcal{N}\left\langle\langle ^ { \bullet } \mathrm { T } \rangle \left\langle\left\langle_{\bullet} \mathrm{B}\right\rangle /[\boldsymbol{D E}]\left\langle\nu_{\bullet}\right\rangle^{\mathrm{K}}\right.\right. \\
\left\langle{ }^{\bullet} \mathrm{T}\right\rangle / /\left[\left\langle\bullet{ }_{\bullet} \mathrm{B}\right\rangle\left\langle\mathrm{B}, \mathrm{B}_{\bullet}\right\rangle\left\langle\mathrm{T}^{\bullet}\right\rangle^{\mathrm{RAI}}\right.
\end{array}}{\boldsymbol{A} / \mathcal{N} /[\boldsymbol{D E}]\left\langle\nu^{\bullet}\right\rangle} \quad \text { RAI }>=\mathrm{K}+\text { ARET }
\end{aligned}
$$

Figure 8: Some application rules [weak interpretation]

We have also tried the parsers on "pathological" sentences, keeping the same grammar. We have considered 4 kinds of recursive constructions indexed by some integer $n$ and used them to build larger and larger sentences (and getting more and more ambiguities). Table 4 shows that the weak interpretation remains faster than the strong one (and it holds true for all the values of $n$ that we have tried).

\begin{tabular}{|l|r|r|r|r|}
\hline & \multicolumn{2}{|c|}{ Strong } & \multicolumn{2}{c|}{ Weak } \\
\hline sentence & TD & Hyb. & TD & Hyb \\
\hline$(\ldots$ of $) 10$ & 6337 & 5362 & 1560 & 1449 \\
\hline (who ... 9 ) & 66138 & 44359 & 13722 & 11808 \\
\hline (who ... 5 (... of) 5 & 25070 & 16922 & 5298 & 4173 \\
\hline (who ... (.. of 3 ) 3 & 43313 & 34602 & 9283 & 8448 \\
\hline
\end{tabular}

Table 4: Results (in ms) for "pathological" sentences

Of course, we are aware that some "pathological" grammars may give better results with the strong interpretation. For example, for the grammar of Figure 10 which generate language $a^{\star}$, we get the results of Figure 11. Now the question is to know if linguistic grammars and sentences exhibit this kind of behaviour.

\section{Conclusion}

We have presented a new Dynamic Programming interpretation of 2SAs specialized to build simple and efficient tabular parsers for TAGs. The preliminary experiments done on a small but realistic linguistic grammar have justified the interest of our approach, even if the optimal worst-case complexity is not en-

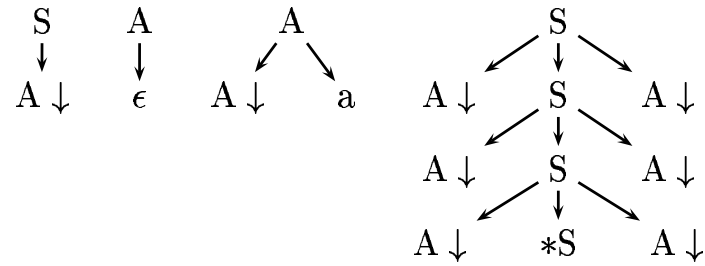

Figure 10: Pathological grammar

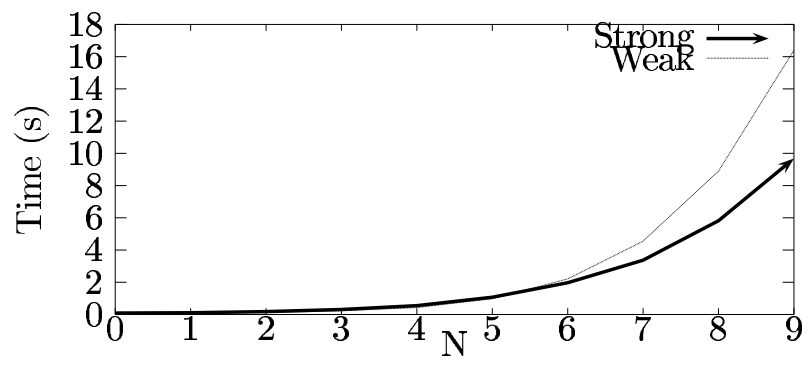

Figure 11: Results for the "pathological" grammar

sured w.r.t. the length of the input string. Of course, more experiments remain to be conducted on a wider range of grammars.

Beside efficiency, the other advantages of our interpretation are its simplicity and the predominance of the notion of continuation. We feel that these ideas could be fruitful in order to design simple tabular parsers satisfying the valid prefix property for other formalisms than TAGs and still running in polynomial time (for instance for mildly contextsensitive formalisms or Linear Context Free Rewrit- 


\begin{tabular}{|l|r|r|r|r|}
\hline Sentence & \multicolumn{2}{|c|}{ Strong } & \multicolumn{2}{|c|}{ Weak } \\
\hline & TD & Hyb. & TD & Hyb \\
\hline$\emptyset$ & 443 & 428 & 250 & 253 \\
\hline Jean aime Marie & 745 & 470 & 124 & 73 \\
\hline Yves montre des fleurs à Sharon & 1494 & 798 & 169 & 97 \\
\hline Kathy regarde un livre avec Stéphane & 1057 & 805 & 157 & 99 \\
\hline un ami américain de Yves qui visite la France boit du bon vin & 2440 & 1594 & 427 & 299 \\
\hline la jolie fille pense que Andrew est le grand ami de son chien & 1679 & 1249 & 274 & 167 \\
\hline Marie comprend que Kathy dort bien chez Andrew & 1326 & 1011 & 241 & 117 \\
\hline Anne voit les fleurs que Yves cherche sur la table & 1851 & 1322 & 278 & 186 \\
\hline Jean pense que Béatrice perd les pédales & 1396 & 1012 & 221 & 130 \\
\hline le petit chat de Sabine court vite & 962 & 721 & 183 & 106 \\
\hline Andrew court parfois les filles & 856 & 674 & 130 & 80 \\
\hline la fille de Marie aime bien les fleurs & 1179 & 896 & 183 & 103 \\
\hline qui est Bonnie & 832 & 529 & 117 & 79 \\
\hline qui dit que Yves a un chien & 1215 & 910 & 188 & 112 \\
\hline un enfant intelligent de Marie comprend lentement que sa mère est bête & 2140 & 1349 & 330 & 200 \\
\hline
\end{tabular}

Table 3: Results (in ms)

ing Systems). In particular, reasoning in terms of continuations and suspensions seems interesting when parsing interleaved constituents (even if the pattern of suspensions is more complex than the one used for TAGs).

It is also interesting to note (once again) that tabulation and parsing strategies are (largely) orthogonal issues: for a parsing strategy expressed by a set of 2SA transitions, we have at least two ways to interpret it using tabulation. Beside the original "strong" interpretation and our "weak" one, there actually exists a whole range of intermediary interpretations corresponding to different levels of tabulation. For instance, by increasing tabulation and using items of the form $\boldsymbol{A} /\left\langle{ }^{\bullet} \mathrm{T}\right\rangle\left\langle{ }_{\bullet} \mathrm{B}\right\rangle /[\boldsymbol{D E}]\left\langle\nu_{\bullet}\right\rangle$, the weak DP interpretation becomes very similar to the Earley prefix-valid algorithm of (Schabes, 1991), with time complexity $O\left(n^{9}\right)$ and space complexity $O\left(n^{6}\right)$. Again, increasing tabulation and using items $\boldsymbol{A} /\left\langle{ }_{\bullet} \mathrm{B}\right\rangle /[\boldsymbol{D E}]\left\langle\nu_{\bullet}\right\rangle$ leads to a DP interpretation close to the strong one but without keeping information relative to the Auxiliary Stack AS in the items. The strong DP interpretation may also be relaxed by not using some projections or the intermediary object $L$ (Figure 5 ). There is also the possibility not to check subsumption on some objects. Some of these possibilities should be investigated, noting that, with some care, several of them can be made to coexist in a single parser.

\section{References}

Miguel Alonso Pardo, Djamé Seddah, and Éric Villemonte de la Clergerie. 2000. Practical aspects in compiling tabular TAG parsers. In Proceedings of the $5^{\text {th }}$ International Workshop on Tree Adjoining Grammars and Related Formalisms (TAG+5), pages 27-32, Université Paris 7, Jussieu, Paris, France.

Tilman Becker. 1994. A new automaton model for TAGs: 2-SA. Computational Intelligence, 10(4).

J. Carroll, N. Nicolov, M. Smets, O. Shaumyan, and D. Weir. 1998. Grammar compaction and computation sharing in automata-based parsing. In Proceedings of Tabulation in Parsing and Deduction (TAPD'98), pages 16-25, Paris (FRANCE).

Roger Evans and David Weir. 1998. A structuresharing parser for lexicalized grammars. In proc. of COLING-ACL'98, pages 372-378.

Aravind K. Joshi. 1987. An introduction to tree adjoining grammars. In Alexis Manaster-Ramer, editor, Mathematics of Language, pages 87115. John Benjamins Publishing Co., Amsterdam/Philadelphia.

Yves Schabes. 1991. The valid prefix property and left to right parsing of tree-adjoining grammar. In Proc. of II International Workshop on Parsing Technologies, IWPT'91, pages 21-30, Cancún, Mexico.

The XTAG Research Group. 1995. A lexicalized tree adjoining grammar for English. Technical Report IRCS 95-03, Institute for Research in Cognitive Science, University of Pennsylvania, 3401 Walnut ST., Suite 400C, Philadelphia, PA 191046228 , March.

Eric Villemonte de la Clergerie and Miguel A. Alonso Pardo. 1998. A tabular interpretation of a class of 2-stack automata. In Proc. of ACL/COLING'98, August. 